\title{
Retrieval of Significant Wave Height and Mean Sea Surface Level Using the GNSS-R Interference Pattern Technique: Results from a three-month Field Campaign
}

\author{
A. Alonso-Arroyo, Student Member, IEEE, A. Camps, Fellow, IEEE, H. Park, Member, IEEE, D. Pascual, Student
} Member, IEEE, R. Onrubia, Student Member, IEEE, and F. Martín, Student Member, IEEE

\begin{abstract}
Since 1993, when the European Space Agency (ESA) proposed the use of GNSS reflected signals for sea mesoscale altimetry, a wide range of applications have appeared. This work focuses on the retrieval of Significant Wave Height (SWH) and the Mean Sea Surface Level (MSSL) from a ground-based experiment using the Interference Pattern Technique (IPT). Two different observables on the IPT are analyzed: the oscillation frequency, and the angle where coherency is lost. The point where coherency in the reflection process is lost can be related to the Rayleigh criterion for smooth surfaces and helps to determine the SWH. Spectral analysis on the interference pattern helps to determine the MSSL. A three-month field campaign was performed on the "Pont del Petroli" pier, Badalona, Spain, to see how the reflected GNSS signals were affected by coastal sea state, and check previous assumptions. Results from this field experiment are shown confirming that the SWH can be retrieved with an accuracy of $6 \mathrm{~cm}$ and the MSSL with $4 \mathrm{~cm}$. Estimations of both parameters are obtained every 30 minutes approximately.
\end{abstract}

Index Terms-GNSS-R, Interference Pattern Technique, Sea Mean Surface Level, Sea Wave Height, Rayleigh criterion

\section{INTRODUCTION}

$\mathbf{N}$ OWADAYS global change is receiving an increasing interest. The rise of temperatures on the Earth has several consequences that have been under rigorous study in the past years. These consequences include diverse areas such as weather patterns, ecology, sea life and marine biodiversity, the inter-tidal zone, impacts on food supply, sea level and sea temperature rising, ocean currents and ocean acidification, glacial and polar cap melting, and coastline degradation [1]. Among them, it is the sea level rising, which is of high concern in different countries, e.g. the Netherlands, where a part of its country is under the mean sea level, and for many densely populated coastal areas of the world [2]. During the last 40 years, the mean sea-level has increased an average of 1.6

The authors are with the Department of Signal Theory and Communications, Universitat Politècnica de Catalunya - BarcelonaTech (UPC), Barcelona Spain, e-mail: alberto.alonso.arroyo@tsc.upc.edu.

These activities have been sponsored by the Spanish Ministry of Science and Innovation, "AROSA-Advanced Radio Ocultations and Scatterometry Applications using GNSS and other opportunity signals", code AYA201129183-C02-01/ESP.

The authors would like to thank the support and help of LIM/UPC, which is the center that manages and maintains the "Pont del Petroli" pier, and in particular the Servei d'Infraestructura Tècnica (SITEC), which manages its experimental activities. $\mathrm{mm} /$ year [3], and $17 \mathrm{~cm}$ in the last century. According to the Intergovernmental Panel on Climate Change (IPCC), the main contributor to the sea level rise is thermal expansion, which is highly related to global warming [4], and ice melting from glaciers, Greenland and the North and South Poles.

Different techniques and technologies are currently being used to monitor the mean sea level [5]. Conventional tide gauges must be in contact with the water and provide point information time series of the mean sea level. A postprocessing stage, which includes daily averaging, provides the mean sea level information. Other systems are based on the observations from different buoys around the world. Buoys capture sea movements, and different characteristics from it can be retrieved afterwards. On the other hand, remote sensing techniques, generally based on satellite platforms containing radar altimeters, are used to measure the sea mean level on a global scale.

In 1993, European Space Agency (ESA) proposed the PAssive Reflectometry and Interferometry System (PARIS) concept as an alternative to conventional radar altimeters [6] [8]. It is based on retrieving mesoscale altimetry by analyzing the direct Global Navigation Satellite Systems (GNSS) signals and the reflected ones on the ocean as well. As there are currently more than 60 GNSS satellites in orbit, and more than 150 are expected by 2020 , data from different reflection points can be acquired at the same time providing an exceptional spatial-temporal sampling. In 1988, the first multistatic techniques for Earth Observation were proposed [9]. Later, in 1991, a French military aircraft testing a Global Positioning System (GPS) receiver got accidentally locked to the GNSS reflected signals on the ocean, leading to a mistaken navigation solution [10]. This proved the feasibility of sensing GNSS reflected signals from an airborne platform. Several remote sensing applications of GNSS reflected signals on different kind of surfaces (i.e., land, water or ice) have appeared [11] [13], including the retrieval of the Mean Sea Surface Level (MSSL), also known as a GPS tide gauge.

Up to present, two different GNSS-Reflectometry (GNSS-R) techniques have been applied in the GPS or GNSS tide gauge known as the SNR-analysis method [14], [15], and the phase-delay analysis method [16], [17]. None of them is able to retrieve the Significant Wave Height (SWH) parameter. A different GNSS-R approach known as 
the Interferometric Complex Field (ICF) has shown before promising results in the estimation of the SWH [18]. This manuscript presents an improved method to retrieve the MSSL or tides, based on the Interference Pattern Technique (IPT), and the retrieval of SWH from the same Signal to Noise Ratio (SNR) observable. This work is divided in 6 different sections. Section II presents the scattering geometry and properties for a low-height ground-based instrument, and the main properties of the IPT. Section III presents the instrumentation used and the field experiment site. Sections IV and $\mathrm{V}$ present the proposed retrieval algorithm for the SWH and the MSSL respectively. Finally, section VI summarizes the conclusions of this work.

\section{GEOMETRY AND THEORETICAL ANALYSIS}

The SNR-analysis method consists of a Right Hand Circular Polarization (RHCP) antenna looking to the zenith acquiring the GNSS direct signal. Due to the sidelobes of the antenna pattern, and not such a good cross-polar ratio out of the antenna beamwidth, for low grazing angles, multipath is observed in the received power or SNR [19]. This multipath is seen as a low frequency modulation in the real data whose fundamental oscillation frequency depends on the antenna height [20], [21]. A change in the sea surface level is seen as a change in the fundamental oscillation frequency. This SNRanalysis method was also previously used for the determination of soil moisture and snow depth from GPS geodetic receivers [22], [23].

The phase-delay analysis method consists of two antennas, one zenith-looking for the direct RHCP GNSS signals, and one nadir-looking for the reflected Left Hand Circular Polarization (LHCP) GNSS signals [24]. The reflected signal travels an additional path, that can be measured using carrier phase measurements and pseudo-range information from the GNSS receivers. A change in the sea surface level is seen as a change in the reflected pseudo-range information. Taking into account the vertical baseline between direct and reflected GNSS antennas, the changes in the sea surface level can be tracked.

These two geometrical configurations have been widely used, but can lead to misinterpretation of the data. In a zenith/nadir-looking antenna, GNSS signals can come from any direction. This means that not only the direct and reflected signals on the surface under study may reach the antenna, also undesired multi-path from other directions, which may distort the shape of the SNR pattern. This undesired multi-path may be difficult to eliminate as the antenna pattern from geodetic antennas tends to be rotationally symmetric. To overcome this problem, this work proposes to use the conventional IPT approach [25]-[27], where a linearly polarized antenna is looking to the horizon instead of the zenith.

\section{A. The IPT over rough surfaces}

The conventional IPT approach was previously tested for water level measurements in a small reservoir $(200 \times 100 \mathrm{~m})$ showing an excellent performance against the ground-truth information. In this approach, undesired reflections coming

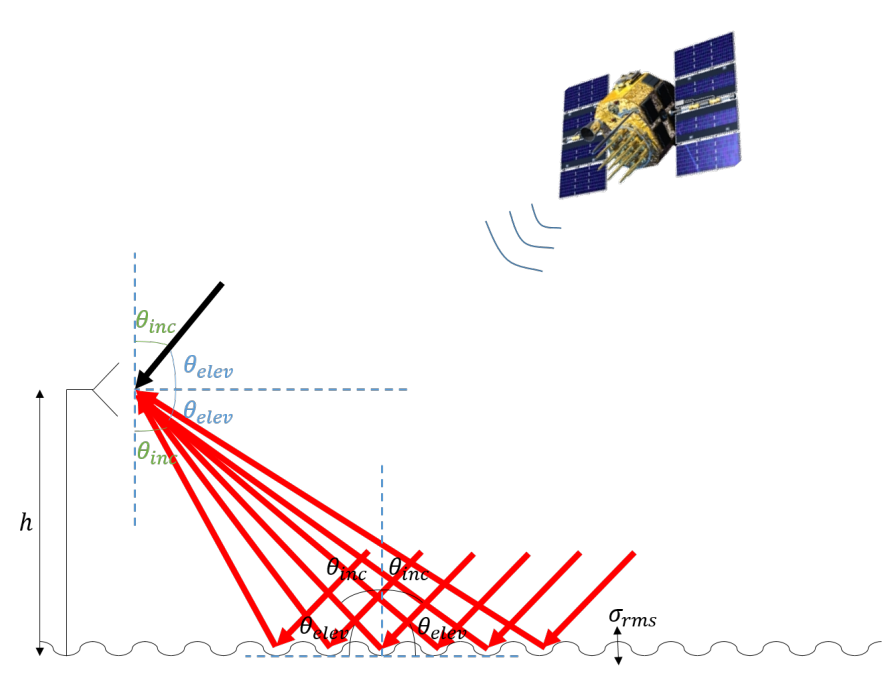

Fig. 1: IPT geometry over a rough surface.

from any direction away of the surface under study are attenuated by the antenna pattern itself, better preserving the shape of the interference pattern [27]. Figure 1 presents the conventional IPT geometrical configuration. It shows in black the direct signal, and in red the reflected signals. In Fig. $1, h$ is the vertical distance between the phase center of the antenna and the reflection surface, $\theta_{i n c}$ is the incidence angle over the mean sea surface, $\theta_{\text {elev }}$ is the elevation angle of the GNSS satellites or the complementary angle of $\theta_{i n c}$, and $\sigma_{h}$ the root mean square $(r m s)$ surface roughness.

Under the horizon-looking geometry shown in Fig. 1, the received power $\left(P_{R}\right)$ at the receiver position is given by:

$$
\begin{aligned}
P_{R} & \propto\left|E_{i}+E_{r}\right|^{2}=\left|E_{0_{i}}\right|^{2} \cdot \mid F_{n}\left(\theta_{\text {elev }}, \phi_{\text {elev }}\right)+ \\
& +\left.\sum_{m=1}^{M} F_{n}\left(\theta_{m}, \phi_{m}\right) A_{m} e^{j \Phi_{m}} e^{j \frac{4 \pi h_{m}}{\lambda} \sin \left(\theta_{m}\right)}\right|^{2},
\end{aligned}
$$

where, $E_{i}$ is the incident electric field, $E_{r}$ the reflected electric field over many scatterers, $E_{0_{i}}$ the incident electric field amplitude, $F_{n}$ the antenna radiation pattern, $\theta_{\text {elev }}$ and $\phi_{\text {elev }}$ the elevation and azimuth of the GNSS satellite respectively, $\lambda$ the wavelength (i.e. $19 \mathrm{~cm}$ for GPS L1-Band), $m$ the scatterer's index, $M$ the total number of scatterers, $\theta_{m}$ and $\phi_{m}$ are the local elevation and azimuth angles of the $\mathrm{m}^{\text {th }}$ scatterer, $A_{m}$ is the $m^{t h}$ scatterer amplitude, and $\Phi_{m}$ the $m^{\text {th }}$ scatterer phase. So, $F_{n}\left(\theta_{\text {elev }}, \phi_{\text {elev }}\right)$ is related to the direct signal whereas $\sum_{m=1}^{M} F_{n}\left(\theta_{m}, \phi_{m}\right) A_{m} e^{j \Phi_{m}} e^{j \frac{4 \pi h_{m}}{\lambda} \sin \left(\theta_{m}\right)}$ to the reflected signal. In the reflected signal, the term $e^{j \frac{4 \pi h_{m}}{\lambda}} \sin \left(\theta_{m}\right)$ is due to the extra path traveled by the signal reflected over the $\mathrm{m}^{\text {th }}$ scatterer with respect to the direct, whereas $F_{n}\left(\theta_{m}, \phi_{m}\right) A_{m} e^{j \Phi_{m}}$ is related to the surface conditions and the antenna pattern. In terrains such as snow or bare-soil, and considering a rotationally symmetric antenna pattern, the reflection can be considered specular [25]-[27], which simplifies the whole reflected term to $R(\theta, \phi) e^{j \phi_{R}}$, where $R$ 
is the Fresnel reflection coefficient and $\phi_{R}$ is its phase at the specular reflection point. If the scattering surface is the sea, the result of the reflected signal term depends strongly on the surface conditions, which defines if the reflection is dominated by the specular component (calm sea) or by the diffuse one (rough sea).

\section{B. Specular and diffuse scattering}

The scattering process has been analyzed by several authors, developing different models depending on the surface conditions (i.e. [28]). In general, the reflected signal has a specular component and a diffuse one. The specular component dominates when the surface is "smooth" enough. When the specular component dominates, the reflection process can be modeled by multiplying the incident wave by the Fresnel reflection coefficient amplitude and phase, and an attenuation factor that depends on the surface's roughness. The diffuse or incoherent component dominates when the surface is "rough" enough. In this case, the scattering coefficient and its phase is different for each scatterer. The total reflected power is the power of the sum of the electric fields coming from each scatterer, resulting in a random amplitude, lower than when the coherent component dominates, and a random phase.

A widely used criterion to differenciate between smooth and rough surfaces is the "Rayleigh criterion" [28]. A surface is considered smooth if:

$$
\sigma_{r m s}<\frac{\lambda}{8 \sin \left(\theta_{\text {elev }}\right)},
$$

which means that the phase difference between all the statterers is lower than $\pi / 2$. More restrictive criteria have been proposed by replacing the factor 8 in Eqn. (2) by 16 or 32 [28], which means that the maximum phase difference between all the scatterers is $\pi / 4$ or $\pi / 8$, respectively. In Eqn. (2) it is seen that, for the same surface's roughness conditions, whether the surface is smooth or rough depends on the electromagnetic wavelength and the incidence angle. The larger the incidence angle or the lower the elevation angle, the smoother the surface appears to be and vice versa. Hence, the lower the elevation angle, the higher the contribution of the coherent component will be as compared to the incoherent one.

Other models were presented trying to improve the accuracy of the "Rayleigh criterion" depending on the observation surface characteristics. Assuming that the sea surface height can be modeled spatially as a 2-D random Gaussian stochastic process [29], Beckmann and Spizzichino computed the mean scattering coefficient [28] under the assumption of the Kirchoff approximation as:

$$
<\rho \rho^{*}>=<\rho><\rho^{*}>+\operatorname{Var}\{\rho\},
$$

where $\rho$ stands for the reflection coefficient in a perfectly conductive surface without shadowing, or multiple scattering. Equation (3) is the second order moment of the reflection coefficient and separates the coherent component $\left(<\rho><\rho^{*}>\right)$ from the incoherent component $(\operatorname{Var}\{\rho\})$. In Eqn. (3) the coherent component is described by Eqn. (4),

$$
<\rho>=\rho_{0} e^{-g / 2},
$$

where $\rho_{0}$ is the reflection coefficient of a smooth perfectly conductive surface without shadowing in all the scattering directions, which vanishes away from the specular reflection direction, and the $g$ factor:

$$
g=\left(\frac{4 \pi \sigma_{r m s} \sin \left(\theta_{\text {elev }}\right)}{\lambda}\right)^{2},
$$

represents how rough is the surface with respect to the wavelength. For $g<<1$ the surface is considered smooth, and for $g>>1$ the surface is considered rough. In Eqn. (3) the incoherent component is given by Eqn. (6):

$$
\operatorname{Var}\{\rho\}=\frac{\pi T^{2} F^{2} e^{-g}}{A} \sum_{m=1}^{\infty} \frac{g^{m}}{m ! m} \cdot \exp \left(-\frac{u_{x y}{ }^{2} T^{2}}{4 m}\right),
$$

where $T$ is the correlation length of the surface, $A$ is the scattering area, $F$ is given by Eqn. (7):

$$
F=\frac{1+\cos \theta_{i} \cos \theta_{s}-\sin \theta_{i} \sin \theta_{s} \cos \phi_{s}}{\cos \theta_{i} \cdot\left(\cos \theta_{i}+\cos \theta_{s}\right)},
$$

being $\theta_{i}$ the incidence angle, $\theta_{s}$ the scattering angle, $\phi_{s}$ the azimuth scattering angle, and, $u_{x y}{ }^{2}$ is given by Eqn. (8):

$$
u_{x y}^{2}=k^{2}\left(\sin ^{2} \theta_{i}-2 \sin \theta_{i} \sin \theta_{s} \cos \phi_{s}+\sin ^{2} \theta_{s}\right),
$$

being $k$ the wavenumber $(2 \pi / \lambda)$.

Equation (4) indicates that the coherent component vanishes away from the specular reflection and it decreases exponentially with the square of the root mean square roughness. The incoherent component depends on the scattering area, which means that it depends on the receiver's height.

In [30], the above formulation was compared to empirical multi-path data over the sea at $1.575 \mathrm{GHz}$. In the cases of calm sea $(g<<1)$ and rough sea $(g>>1)$ those equations can be simplified. Under calm sea conditions the coherent component dominates because the incoherent component is highly attenuated as $g^{m}$ in the summation (Eqn. (6)) tends rapidly to 0 . Under rough sea conditions the incoherent component dominates as the exponential function (Eqn. (4)) highly attenuates the coherent component.

Nevertheless, when $g \approx 1$ the dominant component must be computed. To do so, the scattering coefficient can be normalized by its variance:

$$
\frac{<\rho \rho^{*}>}{\operatorname{Var}\{\rho\}}=1+\frac{<\rho><\rho^{*}>}{\operatorname{Var}\{\rho\}},
$$

which results in:

$$
\frac{<\rho \rho^{*}>}{\operatorname{Var}\{\rho\}}=1+\frac{\rho_{0}^{2}}{\frac{\pi T^{2} F^{2}}{A} \sum_{m=1}^{\infty} \frac{g^{m}}{m ! m} \cdot \exp \left(-\frac{u_{x y} T^{2}}{4 m}\right)} .
$$

The second term in Eqn. (10) is the relationship between the coherent and incoherent components. When it is larger than 1 the coherent component dominates in front of the 
incoherent component, and vice versa. Remember that, away the specular reflection direction the incoherent component always dominates as $\rho_{0}$ vanishes. However, for the specular reflection direction, which is the case of interest in this work, the relationship between coherent and incoherent components varies depending on the elevation angle, and the roughness conditions.

\section{Sea surface characteristics}

The sea surface is fully described by its directional height spectrum $S(k, \phi)$, which can be derived from time series measurements [31]. However, a simplified description involving fewer parameters can also be used. These parameters are:

- the fundamental wavelength, which is the spatial distance between two consecutive wave crests,

- the fundamental wave period, which is the time between two consecutive wave crests,

- the SWH or $H_{\frac{1}{3}}$, which is the average height of the onethird highest waves, and it can be related to the surface roughness parameter as $H_{\frac{1}{3}} \approx 4 \sigma_{r m s}$, being the $\sigma_{r m s}$ the sea surface height standard deviation [29], [32], and

- the steepness, which is the ratio between the wave height and the wavelength.

Simulations using the "Rayleigh Criterion" and Eqn. (10) for different surface correlation lengths and SWH have been performed in order to see the behavior of both the "Rayleigh criterion" and the Beckmann and Spichizzino model [28] (Fig. 2). Figure 2 represents the cut-off elevation angle up to where the coherent component dominates against the incoherent one. The blue and red dashed lines show these angles computed using the "Rayleigh criterion" (Eqn. (2)) for 8 and 16 coefficients in the denominator, respectively. The solid lines show the same cut-off angles for different surface correlation lengths, from 0 up to 2 meters in steps of $40 \mathrm{~cm}$. The lower the correlation length, the larger the dynamic range of elevation angles where the coherent component dominates. When the SWH is larger than 0.5 meters the dynamic range becomes almost independent on the correlation length. In the same figure the solution provided using Beckmann and Spizzichino [28], is in between the "Rayleigh criterion" for the two lowest restrictions (8 and 16)

Figure 2 gives information of the largest elevation angle up to which the interference pattern will be observed. At the same time, it gives information of the smallest elevation angle where the interference pattern is masked by the incoherent scattering power. The computation of this cut-off angle in the experimental interference patterns will be used later to infer the SWH.

\section{FIELD EXPERIMENT DESCRIPTION}

A three-month field campaign with the SMIGOL instrument [25]-[27], [33] at the "Pont del Petroli", Badalona, Spain was conducted between the end of November 2012 and February 2013. The "Pont del Petroli" is a 250 meters long pier. The SMIGOL instrument was installed near the tip of the

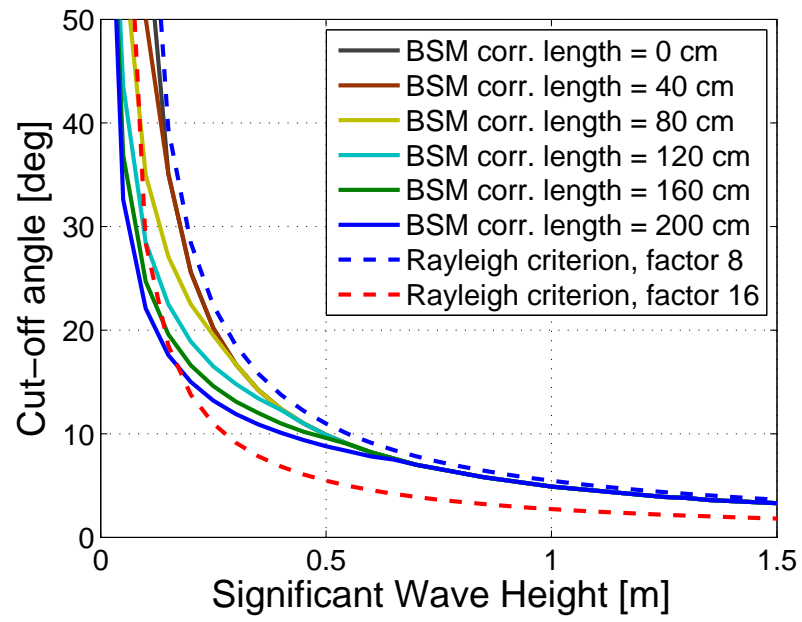

Fig. 2: Comparison between Beckmann and Spizzichino model (BSM) and the Rayleigh criterion. This figure shows the cutoff angle up to when the coherent dominates in front of the incoherent one for both scattering models.

pier, the closest possible to other instruments permanently installed, such as the radar VEGAPULS62, which monitors continuously SWH, the wave period, the mean sea level, and the wind-speed. Figure 3a shows the "Pont del Petroli" top view indicating the location of the SMIGOL reflectometer and the radar VEGAPULS62 on it. The red arrow indicates the SMIGOL instrument antenna pointing direction. Figure $3 \mathrm{~b}$ shows the instrument installed at the pier with the black arrow representing the direct GNSS signals and the red arrow the GNSS reflected signals.

\section{A. The SMIGOL reflectometer}

The SMIGOL reflectometer used in this field experiment is similar to the one used in previous field experiments [25] [27], [33]. It consists of a V-Pol antenna with an azimuth and elevation symmetric pattern, and $90^{\circ}$ antenna beamwidth in both horizontal and vertical planes. After the antenna, there is a Low Noise Amplifier (LNA), and the back-end receiver. The whole system is controlled by a microcontroller that, among other tasks, sets the measurement protocol, which is acquiring GNSS signals for 12 hours, and then 12 hours in idle mode (being this time schedule due to a constraint solely related with the instrument installation specific to this campaign). The power supply was taken from the electrical installation in the pier. The main difference between this SMIGOL version and previous ones is the ruggedized external structure to protect it from harsh environmental conditions, and water splashes.

\section{B. Other instruments at the Pont del Petroli}

The "Pont del Petroli" is a construction devoted to scientific and oceanic research. It has a number of instruments installed permanently to provide continuous meteorological and oceanographic data. For the purpose of this work, only oceanographic data is necessary, specifically the SWH and the MSSL. These data are taken using the radar VEGAPLUS62 which will be the 


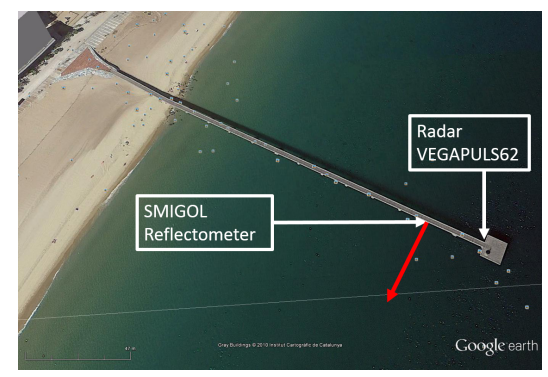

(a) "Pont del Petroli" top view.

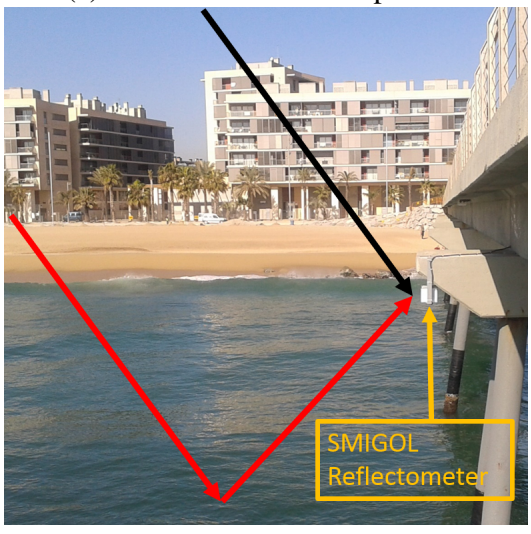

(b) SMIGOL at the "Pont del Petroli".

Fig. 3: Field experiment description: (a) Location and site picture, (b) SMIGOL reflectometer in the horizon-looking geometrical configuration.

sea ground-truth. Figure 4 a shows a picture of it, whereas Fig. $4 \mathrm{~b}$ shows where the instrument is installed and to where the radiation is emitted (nadir-looking). The radar VEGAPULS62 is a high-precision $( \pm 2 \mathrm{~mm}$ ) low-power pulsed K-Band (26 $\mathrm{GHz}$ ) radar with a maximum range of $35 \mathrm{~m}$.

The VEGAPULS 62 measures continuous time-series of the mean sea surface height. From this time-series, the sea wave spectrum is derived (but not stored), from which the main sea descriptors are retrieved. In this work, the main sea descriptors provided by this instrument are compared with the experimental retrieved data.

\section{Significant Wave Height Retrieval}

Figures 5, 6, 7 show three interference pattern samples from the field campaign. Figure 5 shows the interference pattern for GPS satellite 23 on December $4^{\text {th }}$, when the SWH was $20 \mathrm{~cm}$. Figure $5 \mathrm{a}$ shows the raw data retrieved whereas Fig. 5b shows a low-pass filtered version of it to reduce noise. In the low-pass filtered data the coherent component is larger than the incoherent component for GNSS satellite elevation angle $<\sim 30^{\circ}$. At this point, the phase information is lost, as it becomes random, and consequently no more interference pattern is seen for higher elevation angles. Figure 6 shows the interference pattern for GPS satellite 23 on December $6^{t h}$, when the SWH was $62 \mathrm{~cm}$. In there, the coherent component is larger than the incoherent one until an elevation angle of $\sim 11^{\circ}$, when the incoherent becomes

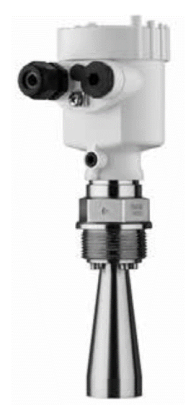

(a) Radar VEGAPULS62 picture.

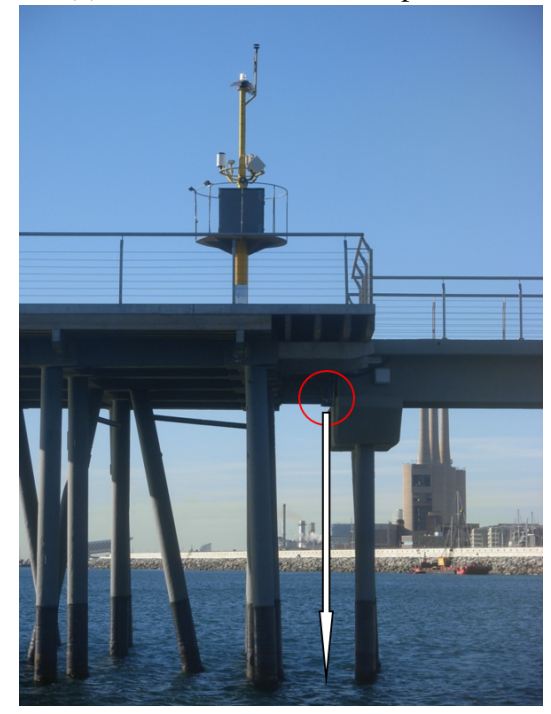

(b) Radar VEGAPULS62 at the "Pont del Petroli".

Fig. 4: Ground-truth instrumentation for measuring oceanographic data, the radar VEGAPULS62.

larger. In those conditions, for higher elevation angles, no more interference pattern is observed. Also, note that the amplitude of the interference pattern oscillations is smaller in Fig. 6 than in Fig. 5, and this is related to the exponential attenuation factor on the coherent component in Eqn. (4). The higher the roughness is, the more attenuated the coherent component is. Also note that, in Fig. 5, the envelope of the interference pattern is increasing. This occurs because the Fresnel reflection coefficient is monotonically increasing after the Brewster angle position ( $\sim 5^{\circ}$ elevation angle), and is not compensated by the surface roughness attenuation.

Figure 7 shows the interference pattern for GPS satellite 23 on December $7^{t h}$, when the SWH was $38 \mathrm{~cm}$, a situation in between December $4^{t h}$ and $6^{t h}$. The elevation angle of the GNSS satellite until the coherent component is larger than the incoherent one is $\sim 17^{\circ}$. It is also in between the previous results, as occurs with the SWH conditions. Also the envelope of the interference pattern is in between them, a fact that also matches with the theoretical aspects shown in section II.

The retrieval of the cut-off angle at which the incoherent component first dominates the coherent one has been performed using overlapped spectrograms [34]. First, as the GNSS satellites can have ascending or descending orbits, the 


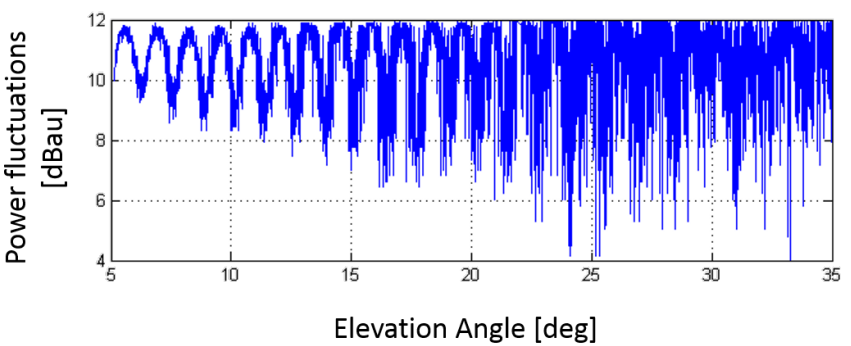

(a) Raw interference pattern.

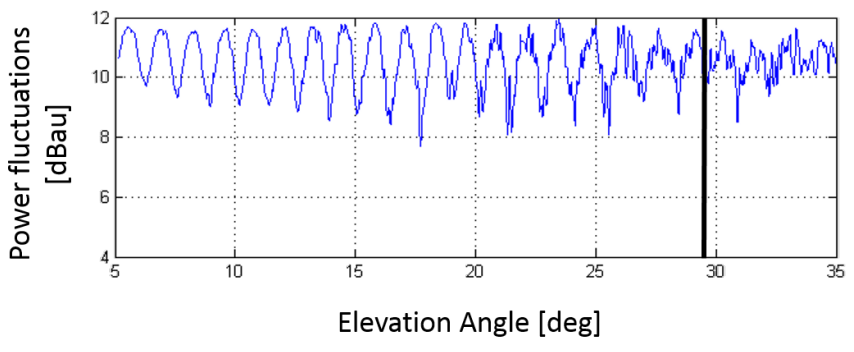

(b) Low-pass filtered interference pattern.

Fig. 5: Interference pattern retrieved for GPS satellite 23 on December $4^{t h}$ and $\mathrm{SWH}=20 \mathrm{~cm}$ measured in $\mathrm{dBau}(\mathrm{dB}$ arbitrary units).

interference patterns are sorted in an ascending elevation angle order. Then, they are split in several consecutive overlapped windows, with a 95\% overlapping factor, to have very fine time-delay resolution. In the interference patterns, time is directly related to the elevation angles. For each of these windows, the Fast Fourier Transform (FFT) is computed in order to create the spectrogram. Then, the fundamental frequency is searched and tracked until its power falls down below a threshold $(20 \mathrm{~dB})$. This point is related to a time value that is directly converted to the corresponding elevation angle. Figures $8 \mathrm{a}$ and $8 \mathrm{~b}$ show the corresponding spectrograms associated to data in Figs. 5 and 6 respectively. The fundamental frequency is marked in black. The spectrogram of Figure $6 \mathrm{~b}$ shows how the fundamental frequency power vanishes due to the loss of coherency in the reflected signal, as opposed to that of Fig. 5b, where the fundamental frequency power does not vanish until almost the end of the spectrogram computation.

This algorithm has been applied to all the interference patterns acquired during the whole three-month field campaign (more than 1000 interference patterns). The cut-off angle computed for each interference pattern has been compared against the SWH parameter delivered by the radar. Figure 9 shows this comparison, the "Rayleigh Criterion" for 8 and 16 factors in the denominator (green and red respectively), and the best fit (Eqn. (11)), which leads to an empirical relation between the estimated cut-off angle and the SWH parameter. From Fig. 9 two main aspects should be remarked. First, due to antenna beamwidth $\left(90^{\circ}\right)$, the interference pattern shape can be only retrieved for elevation angles lower than $45^{\circ}$, so $\mathrm{SWH}$ $\leq 10 \mathrm{~cm}$ cannot be measured. Second, the receivers have an elevation mask, so $\theta_{\text {elev }} \leq 5^{\circ}$ cannot be seen. This makes

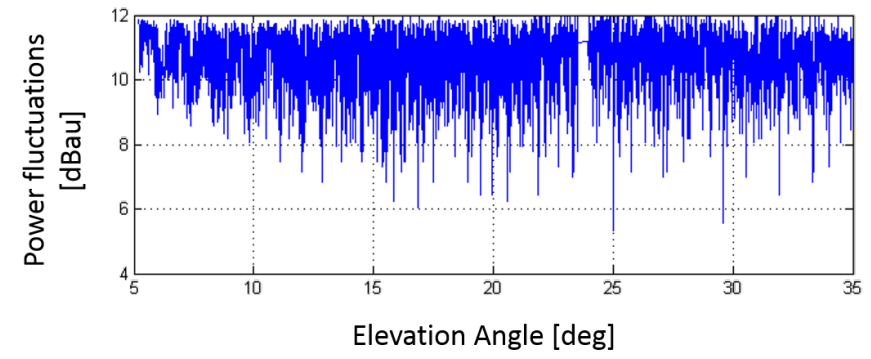

(a) Raw interference pattern.

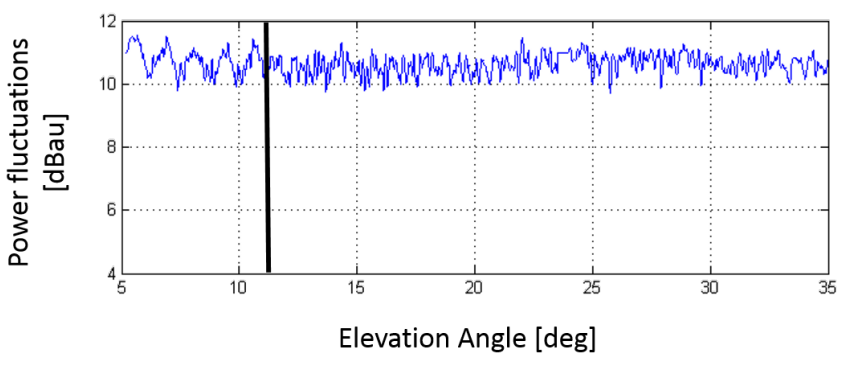

(b) Low-pass filtered interference pattern.

Fig. 6: Interference pattern retrieved for GPS satellite 23 on December $6^{\text {th }}$ and $\mathrm{SWH}=62 \mathrm{~cm}$.

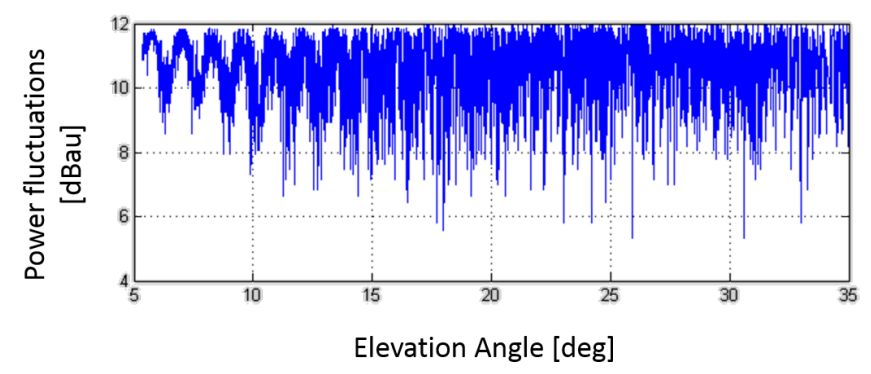

(a) Raw interference pattern.

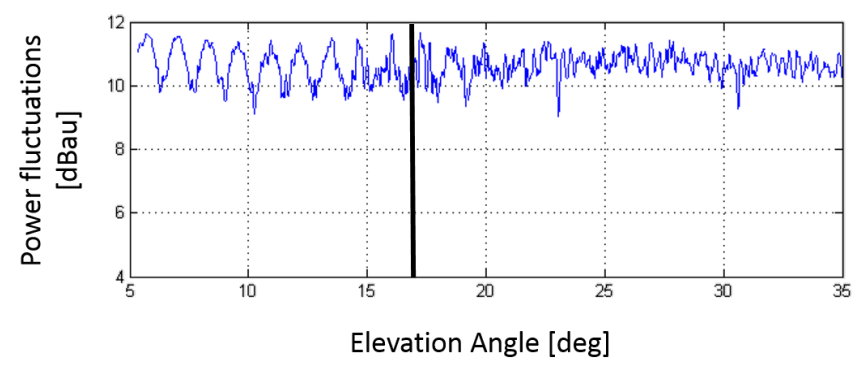

(b) Low-pass filtered interference pattern.

Fig. 7: Interference pattern retrieved for GPS satellite 23 on December $7^{\text {th }}$ and $\mathrm{SWH}=38 \mathrm{~cm}$.

this technique saturate for SWH higher than $70 \mathrm{~cm}$, as for measuring coherency a minimum of two oscillation cycles are needed. If the antenna height is lower than 3 meters, then the maximum value of SWH that can be estimated will decrease as not enough oscillation cycles will be seen in the coherent region [25]. In order to enlarge this dynamic margin three 


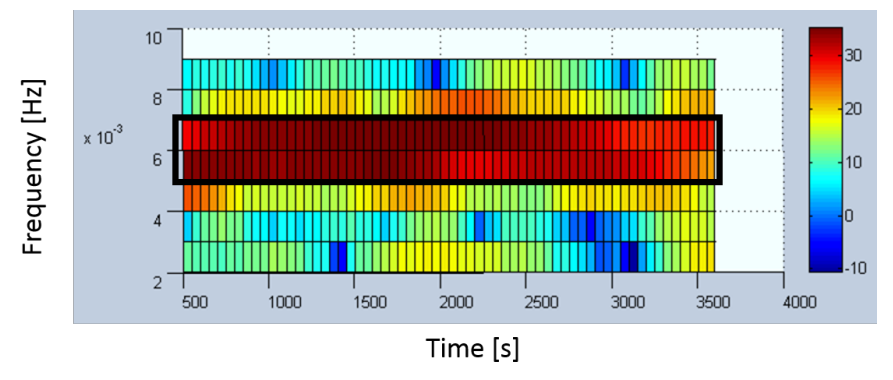

(a) Spectrogram for satellite 23 on December $4^{\text {th }}$

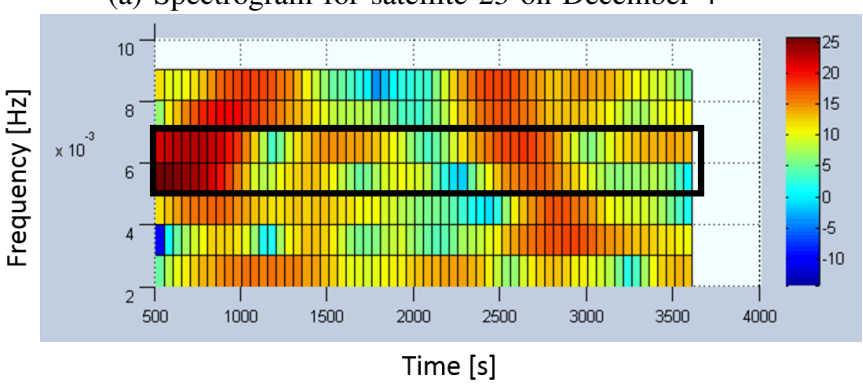

(b) Spectrogram for satellite 23 on December $6^{\text {th }}$

Fig. 8: Spectrograms corresponding to data from Figs. 5 and 6.

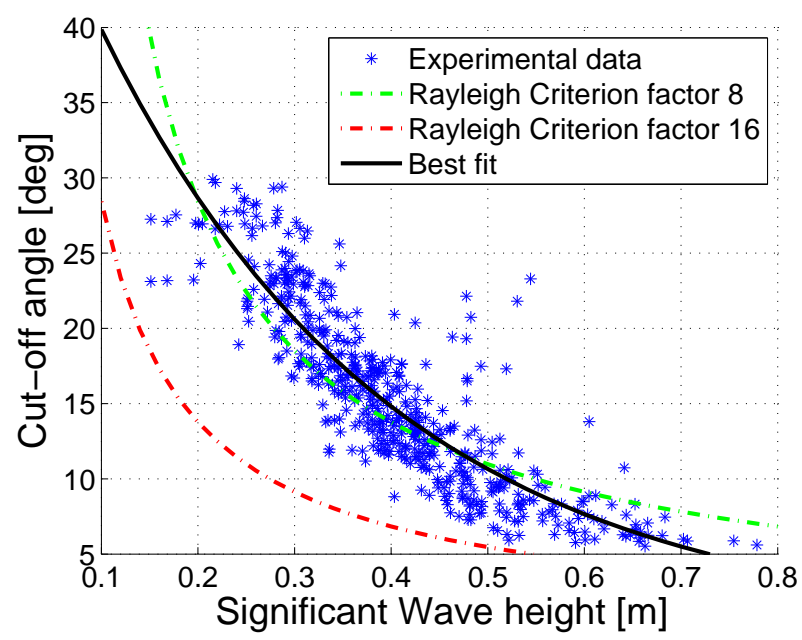

Fig. 9: Comparison between the computed cut-off angle with ground-truth SWH. The best fit has a correlation coefficient of $\mathrm{R}^{2}=0.75$ and a RMSE of $3 .^{\circ}$

different things can be done. First, to measure $\mathrm{SWH} \leq 10$ $\mathrm{cm}$ an antenna with a larger beamwidth is needed. Second, to measure SWH $\geq 70 \mathrm{~cm}$ the SMIGOL instrument should be installed at a higher height, where the oscillation frequency is higher, and then, more oscillations are seen for the same angular region. The maximum height at which the instrument can be installed is limited by the GPS C/A code [25]. The last thing that could be modified is the elevation mask on the back-end receiver.

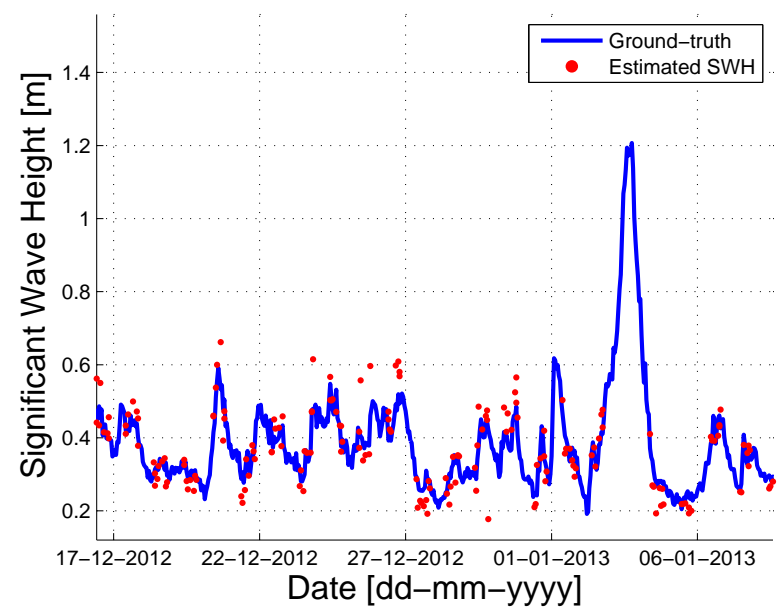

Fig. 10: Comparison between IPT GNSS-R estimated SWH and ground-truth data from December $17^{\text {th }} 2012$ until January $6^{\text {th }}$ 2013. Note that the method performs well up to SWH $<$ $0.7 \mathrm{~m}$, with a correlation coefficient $(\mathrm{R})$ of $90 \%$ in the specified period of time.

$$
\begin{aligned}
& \theta_{\text {cut }- \text { off }} \approx 55.44 \cdot e^{-3.3 \cdot S W H} \\
& \widehat{S W H}=-\frac{1}{3.3} \cdot \ln \left(\frac{\theta_{\text {cut }- \text { off }}}{55.44}\right)
\end{aligned}
$$

By inverting Eqn. (11), the SWH can be estimated (Eqn. (12)). Figure 10 shows 15 days of the SWH evolution in blue and the estimated SWH from the interference patterns (red). The trend of the SWH evolution is followed by the estimated SWH quite precisely except around January $3^{\text {rd }}$, 2013 when SWH is too high. In this region, not enough oscillations were seen in the interference pattern to compute the cut-off angle. Consequently, the SWH could not be retrieved. Figure 11 shows a scatter plot that compares the estimated SWH with the ground-truth SWH from the whole field campaign. In red the $1: 1$ line is added to qualitatively show the correlation between the estimated SWH and the ground-truth information. Analyzing the data from the whole field campaign, an accuracy of $5.7 \mathrm{~cm}$ in the $\mathrm{SWH}$ estimation was achieved for $\mathrm{SWH}$ values lower than 0.7 meters.

\section{MEAN SEA SURFACE LEVEL OR TIDES RETRIEVAL}

From section IV it was seen that only the coherent part of the interference patterns can be used to retrieve geophysical information. This is the part where the reflection coefficient can be modeled as $R e^{j \phi_{R}}$, and the phase term $\left(e^{j \frac{4 \pi h \cos \left(\theta_{\text {elev }}\right)}{\lambda}}\right)$ information is preserved. Equation (1) can be converted into Eqn. (13) considering the proposed approximation, which leads to a similar result previously shown in [21] : 


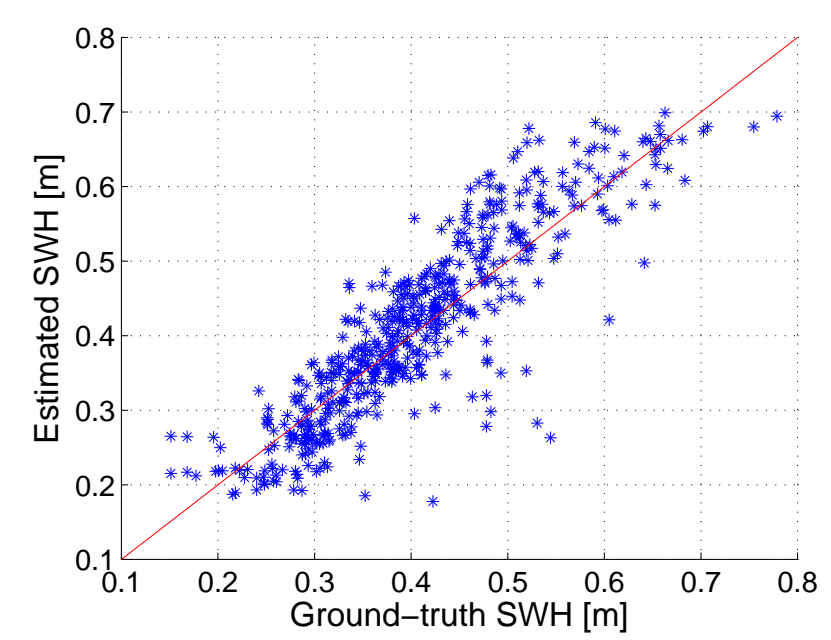

Fig. 11: Comparison between estimated SWH and groundtruth with a correlation coefficient $(\mathrm{R})$ of $86 \%$ for the threemonth field campaign

$$
\begin{aligned}
& P_{R} \propto\left|E_{0_{i}}\right|^{2} \cdot\left(\left|F_{n}\right|^{2}+\left|F_{-n} \cdot R\right|^{2}+\right. \\
& \left.+2 \cdot F_{n} F_{-n} R \cos \left(\frac{4 \pi}{\lambda} h \sin \left(\theta_{\text {elev }}\right)+\phi_{R}+\phi_{F_{-n}}-\phi_{F_{n}}\right)\right),
\end{aligned}
$$

In the previous equation $F_{n}$ stands for $F_{n}(\theta, \phi), F_{-n}$ for $F_{n}(-\theta, \phi), R$ for $R(\theta, \phi), h$ the vertical distance between the antenna phase center and the reflecting surface, and $\theta_{\text {elev }}$ the elevation angle of the GNSS satellite. The fundamental oscillation frequency is identified as $\frac{4 \pi}{\lambda} h \sin \left(\theta_{\text {elev }}\right)$. The other terms are phase terms that may vary slightly this frequency. However, their contribution can be considered negligible as they remain mainly constant along the antenna beamwidth. The phase terms associated to the antenna pattern may vary at most 0.2 $\mathrm{rad}$ in the whole interference pattern acquisition, whereas the value associated to the coherent reflection coefficient phase remains mainly constant. Consequently, the cosine function will be only sensitive to the height variations. Recall that when the incoherent scattering dominates, the reflection coefficient phase is random and this assumption does no longer apply. To estimate the interference pattern fundamental oscillation frequency and infer $h$ it is necessary to apply spectral analysis techniques.

Nevertheless, the oscillation frequency is not constant, as it depends on the $\sin \left(\theta_{\text {elev }}\right)$, and $\theta_{\text {elev }}$ is a function that depends not linearly on time. If a change of variable is performed, the interference pattern can be plotted as a function of the $\sin \left(\theta_{\text {elev }}\right)$ instead of plotting it as a function of $\theta_{\text {elev }}$. Then, the oscillation frequency becomes constant $\left(\frac{2 h}{\lambda}\right)$ if the mean sea surface level does not change during the interference pattern acquisition. This change of variable may result very useful to find the oscillation frequency, but unfortunately the properties of the acquired data change. Before, the interference pattern was regularly sampled at $1 \mathrm{~Hz}$. The change of variable converts the regular sampling pattern to an unevenly sampling pattern and conventional spectral analysis techniques such as FFT no longer apply. Unevenly sampling patterns have been already analyzed in the astronomy field, where it is quite difficult to obtain regularly sampling patterns. Several techniques have been developed in order to analyze periodicity and frequency estimation in that conditions. The most used techniques are the Fourier Periodogram (FP) [35], the LombScargle Periodogram (LSP) or Least-Squares Periodogram (LS) [35]-[38], and the CAPON (CAP) [39]. All of them have been applied to the interference patterns obtained in this field experiment and their accuracy is compared independently.

\section{A. The Fourier Periodogram}

The classical Fourier transform-based periodogram [35] is given by:

$$
P_{F}(w)=\frac{1}{N^{2}}\left|\sum_{n=1}^{N} y\left(t_{n}\right) e^{-j w t_{n}}\right|^{2},
$$

where $w$ is the frequency factor, $N$ the number of samples, $n$ the sample number, and $t_{n}=\sin \left(\theta_{\text {elev }}(n)\right)$ the sampling time. Note that the sample $\left(y\left(t_{n}\right)\right)$ and the sample time $\left(t_{n}\right)$ are intrinsecally related in the equation, consequently this method is suitable for unevenly sampling patterns. Equation (14) comes from a least-squares data fitting problem and it is suitable for complex-value data. However, for the case of real-value data, which is the one used in this work, the LS or LSP are more suitable methods.

\section{B. The Lomb-Scargle Periodogram (LSP)}

The LSP [38] is given by:

$$
P_{L S P}(w)=\frac{1}{\widehat{Y Y}}\left(\frac{{\widehat{Y C_{\hat{\tau}}}}^{2}}{{\widehat{C C_{\hat{\tau}}}}^{2}}+\frac{{\widehat{Y S_{\hat{\tau}}}}^{2}}{{\widehat{S S_{\hat{\tau}}}}^{2}}\right)
$$

where:

$$
\begin{aligned}
& \widehat{Y Y}=\sum_{n=1}^{N} y\left(t_{n}\right)^{2}, \\
& \widehat{Y C_{\hat{\tau}}}=\sum_{n=1}^{N} y\left(t_{n}\right) \cdot \cos w\left(t_{n}-\hat{\tau}\right), \\
& \widehat{C C_{\hat{\tau}}}=\sum_{n=1}^{N} \cos ^{2} w\left(t_{n}-\hat{\tau}\right), \\
& \widehat{Y S_{\hat{\tau}}}=\sum_{n=1}^{N} y\left(t_{n}\right) \cdot \sin w\left(t_{n}-\hat{\tau}\right), \\
& \widehat{S S_{\hat{\tau}}}=\sum_{n=1}^{N} \sin ^{2} w\left(t_{n}-\hat{\tau}\right), \\
& \tan 2 w \hat{\tau}=\frac{\sum_{n=1}^{N} \sin 2 w t_{n}}{\sum_{n=1}^{N} \cos 2 w t_{n}} .
\end{aligned}
$$

Again, every sample its related to its acquisition time so it suitable for both uniform and non-uniform sampling patterns. 


\section{The Least-Squares (LS) Periodogram}

The LS [35] is the least-squares FP for real-valued data and is given by:

$$
P_{L S}(w)=\frac{1}{N} \mathbf{r}^{T}(w) \mathbf{R}^{-1}(w) \mathbf{r}(w)
$$

where:

$$
\begin{aligned}
& \mathbf{R}(w)=\sum_{n=1}^{N}\left[\begin{array}{l}
\cos \left(w t_{n}\right) \\
\sin \left(w t_{n}\right)
\end{array}\right]\left[\begin{array}{ll}
\cos \left(w t_{n}\right) & \left.\sin \left(w t_{n}\right)\right]
\end{array}\right. \\
& \mathbf{r}(w)=\sum_{n=1}^{N}\left[\begin{array}{c}
\cos \left(w t_{n}\right) \\
\sin \left(w t_{n}\right)
\end{array}\right] y\left(t_{n}\right) \text {. }
\end{aligned}
$$

\section{The CAPON (CAP) spectral estimator}

The above periodograms sometimes have leakage or low resolution components due to correlation between different spectral components. The CAP method tries to overcome these problems by estimating the covariance matrix between samples. The CAP spectrum is given by [39];

$$
P_{C A P}(w)=\frac{1}{\mathbf{a}^{H}\left(e^{j w \Delta}\right) \hat{\mathbf{R}}^{-1} \mathbf{a}\left(e^{j w \Delta}\right)},
$$

where:

$$
\begin{gathered}
\mathbf{a}\left(e^{j w \Delta}\right)=\left[\begin{array}{llll}
1 & e^{j w \Delta} & \cdots & e^{j w \Delta m}
\end{array}\right]^{T}, \\
\hat{\mathbf{R}}=\frac{1}{\tilde{N} \Delta} \sum_{p=0}^{\tilde{N}-1} \mathbf{a}\left(e^{j w_{p} \Delta}\right) \mathbf{a}^{H}\left(e^{j w_{p} \Delta}\right) P_{F P}\left(w_{p}\right),
\end{gathered}
$$

which can also be related to the standard covariance matrix,

$$
\Delta=\frac{1}{N-1} \sum_{k=0}^{N-2}\left(t_{k+1}-t_{k}\right)
$$

which is the average sampling period, $m$ the length of the CAP filters, and $\tilde{N} \geq N$ as it is related to the number of spectral components aimed to estimate, which is normally larger than the number of samples.

\section{E. Experimental Results}

All spectral estimators mentioned above have been applied to the three-month field campaign, and their results compared against the ground-truth data from the radar installed at the "Pont del Petroli". Figure 12 shows the spectra for the interference pattern presented on Fig. 5 using the different proposed spectral estimators. The FP, the LSP and the LS provide similar spectral estimations despite an amplitude or normalization term that can be equalized. They coincide in the spectra estimated and in the height retrieval. However, the CAP estimator differs slightly in the spectra and the height retrieval estimation. As seen in Fig. 12d, the CAP estimator tries to reduce the contribution of the sidelobes in the spectra and to improve the accuracy of the fundamental oscillation frequency estimation.
Figure 13 shows the comparison between the estimated SMIGOL height and the ground-truth measured by the radar. Figure 13a compares the results obtained using the FP spectral estimator to retrieve the mean SMIGOL height with the goundtruth mean sea level. Figure 13b shows the results obtained using the LSP spectral estimator. Figure 13c shows the results obtained using the LS. Figure 13d shows the results obtained using the CAP spectral estimator. The FP, the LSP and the LS provide similar results. Conversely, the CAP seems to provide a better estimation as there are less outliers and the points are closer to the best linear fit curve. Table I shows the statistical parameters of the best linear fits in Fig. 13. In the "Best fit" field the linear fit equation is presented where $S H$ stands for the SMIGOL Height, and $M S L$ stands for the the mean surface level. In the $\mathrm{R}^{2}$ the Pearson product-moment correlation coefficient squared is indicated. The RMSE field indicates the fit standard error or root mean square error.

There are two remarkable aspects in Fig. 13 and Tab. I. First, the relationship between the SMIGOL height estimation and the sea surface mean level is negative. This occurs because an increase in the sea surface level means a decrease in the vertical distance between the SMIGOL antenna phase center and the reflection surface, and vice versa. Furthermore, this relationship must be -1 , as $1 \mathrm{~cm}$ increase in sea surface height is equivalent to $1 \mathrm{~cm}$ decrease in the estimated SMIGOL height. The best linear fits shown in Tab. I indicate that this relationship is accomplished. The CAP method seems to give the best performance among them, as the slope of the best linear fit is -1.001 whereas the others are around -0.975 and -0.978 . Second, the FP, the LSP and the LS provide a similar accuracy $(\sim 5 \mathrm{~cm})$ in the MSSL retrieval. Nevertheless, the CAP method provides approximately $\sim 4 \mathrm{~cm}$ accuracy, improving the results obtained with previous estimators.

To use this kind of system as a tide gauge instrument the following steps must be followed:

1) Determine the fundamental oscillation frequency from the interference patterns.

2) Convert the fundamental oscillation frequency to the instrument equivalent height using Eqn. (23).

3) Define a mean sea surface reference level (MSSRL).

4) Determine the actual MSSL with respect to the reference level using Eqn. (24):

$$
\begin{gathered}
H_{e q}=\frac{f_{o s c} \cdot \lambda}{2}, \\
\mathrm{MSSL}=-\left(H_{e q}-\mathrm{MSSRL}\right) .
\end{gathered}
$$

Equation (24) emphasizes the fact that an increase in the actual MSSL is related to a decrease in the observed $H_{e q}$ and vice versa. To end this section, Fig. 14 shows a time series of the estimated MSSL values in red dots and the groundtruth information in a blue solid line in order to validate the proposed algorithm. The same time axis than for Fig. 11 has been used. 


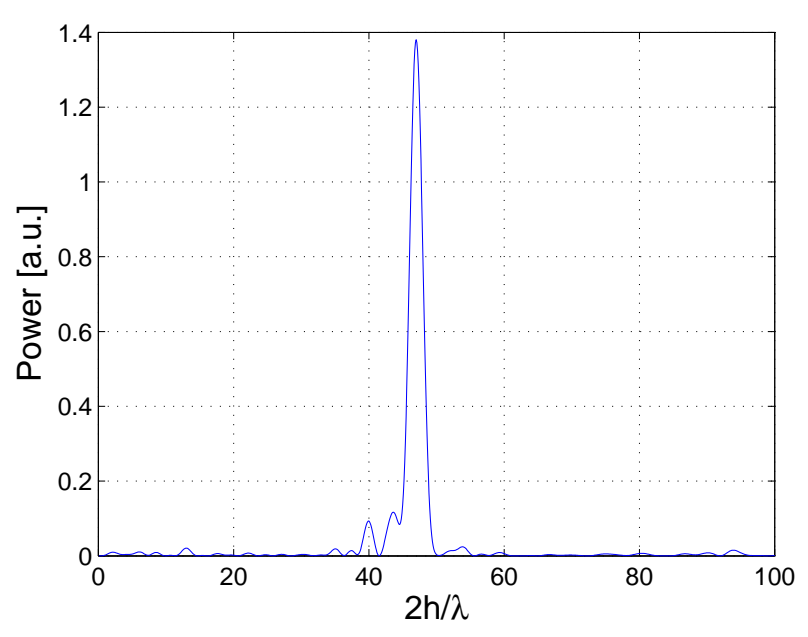

(a) FP for satellite 23 on December $4^{t h}$

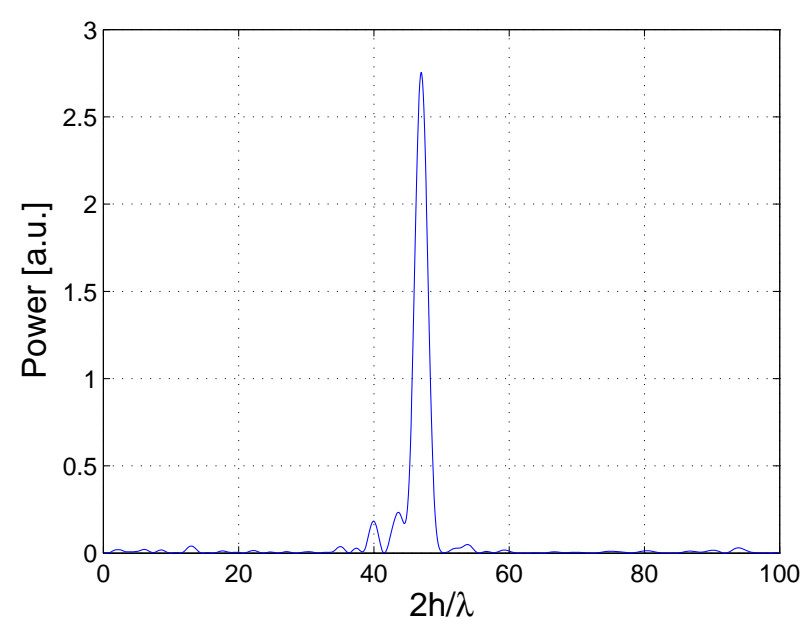

(c) LS for satellite 23 on December $4^{\text {th }}$

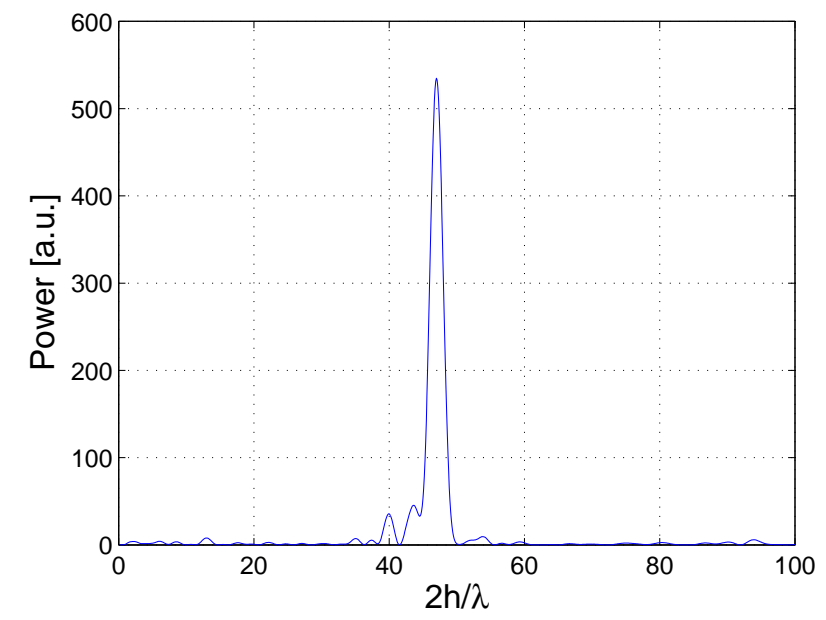

(b) LSP for satellite 23 on December $4^{\text {th }}$

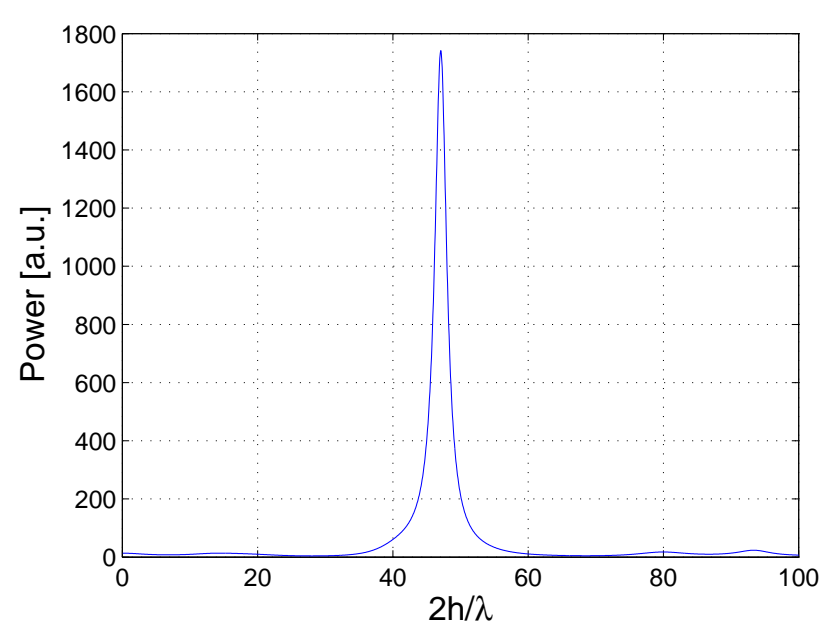

(d) CAP for satellite 23 on December $4^{\text {th }}$

Fig. 12: Spectra for the satellite 23 interference pattern on December $4^{\text {th }}$.

TABLE I: Statistical parameters of the best linear fits in Fig. 13

\begin{tabular}{|c|c|c|c|}
\hline Estimator & Best fit & $\mathrm{R}^{2}$ & RMSE $[\mathrm{cm}]$ \\
\hline \hline FP & $S H=-0.9745 \cdot M S L+4.345$ & 0.695 & 5.08 \\
\hline LSP & $S H=-0.9783 \cdot M S L+4.345$ & 0.698 & 5.06 \\
\hline LS & $S H=-0.9783 \cdot M S L+4.345$ & 0.698 & 5.06 \\
\hline CAP & $S H=-1.001 \cdot M S L+4.333$ & 0.782 & 4.15 \\
\hline
\end{tabular}

\section{CONCLUSIONS}

This work has presented the results of a three-month field campaign using the IPT to retrieve the SWH and MSSL from the observed interference patterns. An accuracy on the SWH estimation of $5.7 \mathrm{~cm}$ and an accuracy of $4.1 \mathrm{~cm}$ on the MSSL is demonstrated using the experimental data. Estimations of both parameters can be obtained every 30 minutes approximately.

The use of a horizon-looking antenna in the IPT attenuates undesired reflections from points away of the reflection surface which helps to preserve better the shape of the interference pattern.

The point where the coherency in the reflection process is lost is used to determine the SWH. At this point, no more interference pattern is seen as the incoherent reflection dominates in front of the coherent one, and the reflected phase becomes random. Using the spectrogram, this point can be easily determined in time and related to the GNSS elevation angle or surface incidence angle. Then, this elevation angle is related to surface roughness. For GPS L1-Band the observed surface roughness may be related to the fundamental 


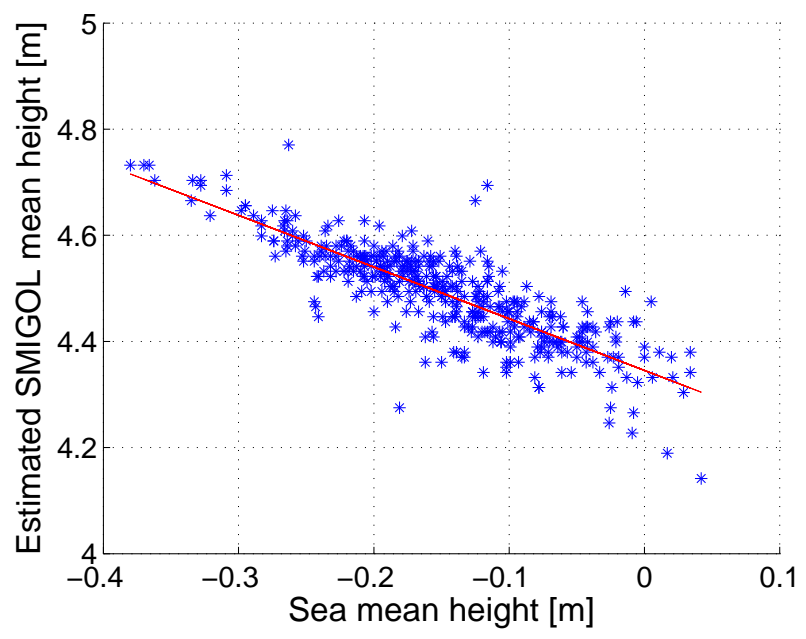

(a) FP.

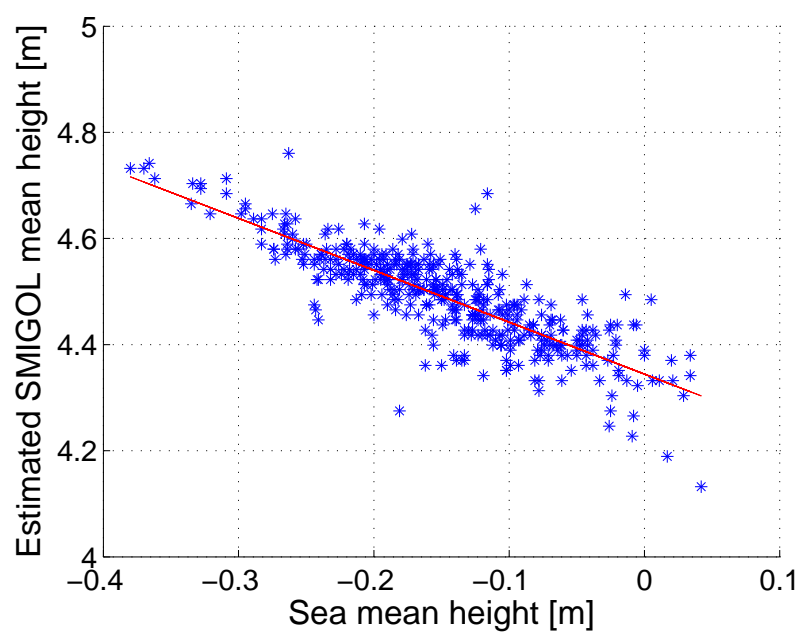

(c) LS.

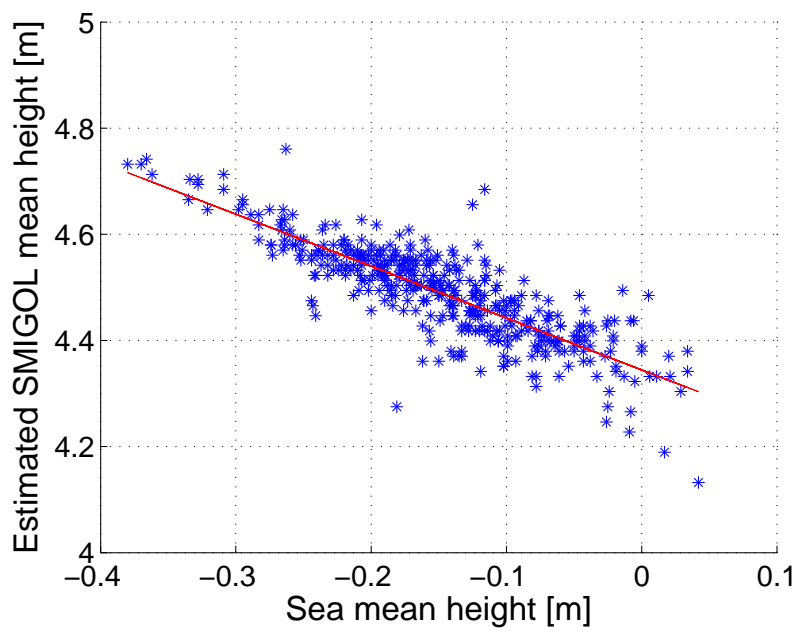

(b) LSP.

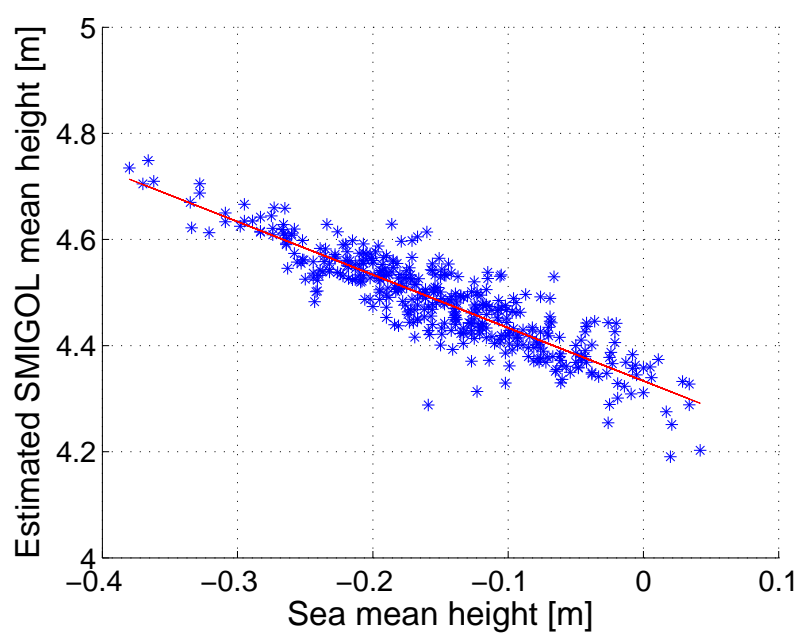

(d) CAP.

Fig. 13: Mean sea surface level retrieval. Comparison between Instrument height estimation and mean sea level ground-truth information. In red the best linear fit.

sea waves. Consequently, surface roughness may be related to the SWH. This technique works for SWH values between 10 $\mathrm{cm}$ and $70 \mathrm{~cm}$. The former $(10 \mathrm{~cm})$ because the interference pattern can only be used for elevation angles lower than $45^{\circ}$ due to antenna pattern beamwidth. The latter $(70 \mathrm{~cm})$ because the SMIGOL's receiver has an elevation angle mask of $5^{\circ}$, so the power fluctuations for satellites whose elevation angle is lower than that cannot be measured. An empirical equation to determine the SWH depending on the cut-off coherency angle has been derived. Empirical data demonstrates that the less restrictive Rayleigh criterion for rough surfaces is the theoretical model that works better to identify the cut-off coherency angle.

To retrieve the MSSL only the coherent part of the interference pattern can be used. It is the part that has one fundamental oscillation frequency. This fundamental oscillation frequency is not constant as it depends on the sine of the GNSS satellite elevation angle. A change of variable is performed to make it constant. As a consequence of this change of variable, spectral techniques for unevenly spaced data must be used to estimate the fundamental oscillation frequency. Different spectral techniques specifically designed for unevenly spaced data have been used to determine the fundamental oscillation frequency. The CAPON method has shown to perform better than the Fourier periodogram, the Lomb-Scargle periodogram, and the Fourier Least-Squares periodogram. The estimated oscillation frequency is then related to the vertical distance between the SMIGOL antenna phase center and the average reflection surface, which is then related to the MSSL using a reference value.

\section{REFERENCES}

[1] T. M. Letcher, Climate Change, T. Letcher, Ed. Elsevier B.V., 2009.

[2] S. Zerbini, T. Baker, M. Negusini, H.-P. Plag, and C. Romagnoli, "Height variations and secular changes in sea level," J. Geodyn. vol. 25, no. 3-4, pp. 241-262, May 1998. 


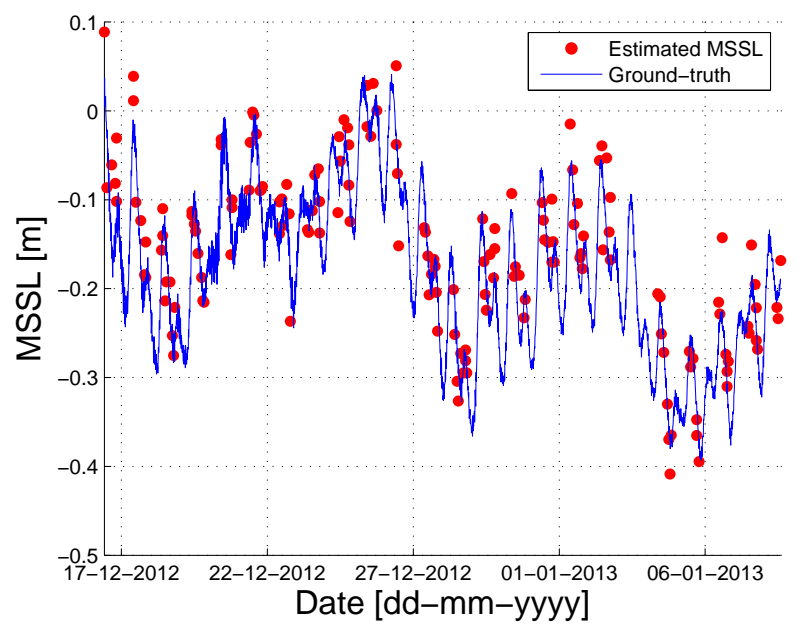

Fig. 14: Comparison between estimated MSSL and groundtruth with a correlation coefficient $(\mathrm{R})$ of $88 \%$.

[3] C. M. Domingues, J. A. Church, N. J. White, P. J. Gleckler, S. E. Wijffels, P. M. Barker, and J. R. Dunn, "Improved estimates of upper-ocean warming and multi-decadal sea-level rise." Nature, vol. 453, no. 7198, pp. 1090-3, Jun. 2008.

[4] T. M. L. Wigley and S. C. B. Raper, "Thermal expansion of sea water associated with global warming," Nature, vol. 330, no. 6144, pp. 127-131, Nov. 1987

[5] Intergovernmental Oceanographic Comission (IOC), "MANUAL ON SEA LEVEL," Tech. Rep., 1985.

[6] M. Martín-Neira, "A passive reflectometry and interferometry system(PARIS): Application to ocean altimetry," ESA J., vol. 17, pp. 331-355, 1993.

[7] M. Martin-Neira, M. Caparrini, J. Font-Rossello, S. Lannelongue, and C. Vallmitjana, "The PARIS concept: an experimental demonstration of sea surface altimetry using GPS reflected signals," IEEE Trans. Geosci. Remote Sens., vol. 39, no. 1, pp. 142-150, 2001.

[8] M. Martin-Neira, S. D'Addio, C. Buck, N. Floury, and R. PrietoCerdeira, "The PARIS Ocean Altimeter In-Orbit Demonstrator," IEEE Trans. Geosci. Remote Sens., vol. 49, no. 6, pp. 2209-2237, Jun. 2011.

[9] C. Hall and R. Cordey, "Multistatic Scatterometry," in Int. Geosci. Remote Sens. Symp. 'Remote Sens. Mov. Towar. 21st Century'. IEEE 1988, pp. 561-562.

[10] J.-M. R. Jean-Claude Auber, Alain Bibaut, "Characterization of Multipath on Land and Sea at GPS Frequencies," in Proc. 7th Int. Tech. Meet. Satell. Div. Inst. Navig. (ION GPS 1994), Salt Lake City, 1994, pp. 1155-1171.

[11] S. Jin and A. Komjathy, "GNSS reflectometry and remote sensing: New objectives and results," Adv. Sp. Res., vol. 46, no. 2, pp. 111-117, Jul. 2010.

[12] S. Jin, G. Feng, and S. Gleason, "Remote sensing using GNSS signals: Current status and future directions," Adv. Sp. Res., vol. 47, no. 10, pp. 1645-1653, May 2011.

[13] E. Cardellach, F. Fabra, O. Nogués-Correig, S. Oliveras, S. Ribó, and A. Rius, "GNSS-R ground-based and airborne campaigns for ocean, land, ice, and snow techniques: Application to the GOLD-RTR data sets," Radio Sci., vol. 46, no. 6, pp. n/a-n/a, Dec. 2011.

[14] K. M. Larson, R. D. Ray, F. G. Nievinski, and J. T. Freymueller, "The Accidental Tide Gauge: A GPS Reflection Case Study From Kachemak Bay, Alaska," IEEE Geosci. Remote Sens. Lett., pp. 1-5, 2013.

[15] K. M. Larson, J. S. Löfgren, and R. Haas, "Coastal sea level measurements using a single geodetic GPS receiver," Adv. Sp. Res., vol. 51, no. 8, pp. 1301-1310, Apr. 2013.

[16] J. S. Löfgren, R. Haas, H.-G. Scherneck, and M. S. Bos, "Three months of local sea level derived from reflected GNSS signals," Radio Sci., vol. 46, no. 6, pp. n/a-n/a, Dec. 2011.

[17] J. S. Löfgren, R. Haas, and J. M. Johansson, "Monitoring coastal sea level using reflected GNSS signals," Adv. Sp. Res., vol. 47, no. 2, pp. 213-220, Jan. 2011.

[18] A. Egido, M. Delas, M. Garcia, and M. Caparrini, "Non-space applications of GNSS-R: From research to operational services.
Examples of water and land monitoring systems," in 2009 IEEE Int. Geosci. Remote Sens. Symp. IEEE, 2009, pp. II-170-II-173.

[19] F. G. Nievinski and K. M. Larson, "Inverse Modeling of GPS Multipath for Snow Depth Estimation-Part I: Formulation and Simulations," IEEE Trans. Geosci. Remote Sens., vol. 52, no. 10, pp. 6555-6563, Oct. 2014.

[20] A. Kavak, W. Vogel, and G. Xu, "Using GPS to measure ground complex permittivity," Electron. Lett., vol. 34, no. 3, p. 254, 1998

[21] V. U. Zavorotny, K. M. Larson, J. J. Braun, E. E. Small, E. D. Gutmann, and A. L. Bilich, "A Physical Model for GPS Multipath Caused by Land Reflections: Toward Bare Soil Moisture Retrievals,' IEEE J. Sel. Top. Appl. Earth Obs. Remote Sens., vol. 3, no. 1, pp. 91-99, Mar. 2010.

[22] K. M. Larson, J. J. Braun, E. E. Small, V. U. Zavorotny, E. D. Gutmann, and A. L. Bilich, "GPS Multipath and Its Relation to Near-Surface Soil Moisture Content," IEEE J. Sel. Top. Appl. Earth Obs. Remote Sens., vol. 3, no. 1, pp. 91-99, Mar. 2010.

[23] K. M. Larson, E. D. Gutmann, V. U. Zavorotny, J. J. Braun, M. W. Williams, and F. G. Nievinski, "Can we measure snow depth with GPS receivers?" Geophys. Res. Lett., vol. 36, no. 17, p. L17502, Sep. 2009.

[24] V. Zavorotny and A. Voronovich, "Scattering of GPS signals from the ocean with wind remote sensing application," IEEE Trans. Geosci. Remote Sens., vol. 38, no. 2, pp. 951-964, Mar. 2000.

[25] N. Rodriguez-Alvarez, X. Bosch-Lluis, A. Camps, M. Vall-llossera, E. Valencia, J. Marchan-Hernandez, and I. Ramos-Perez, "Soil Moisture Retrieval Using GNSS-R Techniques: Experimental Results Over a Bare Soil Field," IEEE Trans. Geosci. Remote Sens., vol. 47, no. 11, pp. 3616-3624, Nov. 2009.

[26] N. Rodriguez-Alvarez, X. Bosch-Lluis, A. Camps, A. Aguasca, M. Vall-llossera, E. Valencia, I. Ramos-Perez, and H. Park, "Review of crop growth and soil moisture monitoring from a ground-based instrument implementing the Interference Pattern GNSS-R Technique,' Radio Sci., vol. 46, no. 6, pp. n/a-n/a, Dec. 2011.

[27] N. Rodriguez-Alvarez, X. Bosch-Lluis, A. Camps, I. Ramos-Perez, E. Valencia, H. Park, and M. Vall-llossera, "Water level monitoring using the interference pattern GNSS-R technique," in 2011 IEEE Int. Geosci. Remote Sens. Symp. IEEE, Jul. 2011, pp. 2334-2337.

[28] P. Beckmann; A. Spizzichino, The scattering of electromagnetic waves from rough surfaces. Artech Print on Demand, 1987.

[29] B. Kinsman, Wind Waves: Their Generation and Propagation on the Ocean Surface. Dover Phoenix Editions, 1984.

[30] Y. Karasawa and T. Shiokawa, "Characteristics of L-band multipath fading due to sea surface reflection," IEEE Trans. Antennas Propag., vol. 32, no. 6, pp. 618-623, Jun. 1984

[31] a.T. Manninen, "Multiscale Surface Roughness and Backscattering Summary," J. Electromagn. Waves Appl., vol. 11, no. 4, pp. 471-475, Jan. 1997.

[32] F. Hogben, N.; Lumb, Ocean Wave Statistics: A Statistical Survey of Wave Characteristics Estimated Usually from Voluntary Observing Ships Sailing Along the Shipping Routes of the World. H.M. Stationery Office, 1967.

[33] N. Rodriguez-Alvarez, A. Camps, M. Vall-llossera, X. Bosch-Lluis, A. Monerris, I. Ramos-Perez, E. Valencia, J. F. Marchan-Hernandez, J. Martinez-Fernandez, G. Baroncini-Turricchia, C. Perez-Gutierrez, and N. Sanchez, "Land Geophysical Parameters Retrieval Using the Interference Pattern GNSS-R Technique," IEEE Trans. Geosci. Remote Sens., vol. 49, no. 1, pp. 71-84, Jan. 2011.

[34] L. Cohen, "Time-frequency distributions-a review," Proc. IEEE, vol. 77, no. 7, pp. 941-981, Jul. 1989.

[35] P. Stoica, "Spectral Analysis of Nonuniformly Sampled Data: A New Approach Versus the Periodogram," IEEE Trans. Signal Process., vol. 57, no. 3, pp. 843-858, Mar. 2009.

[36] N. R. Lomb, "Least-squares frequency analysis of unequally spaced data," Astrophys. Space Sci., vol. 39, no. 2, pp. 447-462, Feb. 1976.

[37] J. D. Scargle, "Studies in astronomical time series analysis. II Statistical aspects of spectral analysis of unevenly spaced data," Astrophys. J., vol. 263, p. 835, Dec. 1982.

[38] M. Zechmeister and M. Kürster, "The generalised Lomb-Scargle periodogram," Astron. Astrophys., vol. 496, no. 2, pp. 577-584, Mar 2009.

[39] P. Stoica and N. Sandgren, "Spectral analysis of irregularly-sampled data: Paralleling the regularly-sampled data approaches," Digit. Signal Process., vol. 16, no. 6, pp. 712-734, Nov. 2006. 


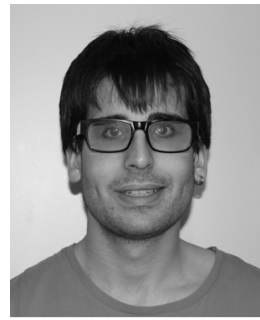

Alberto Alonso Arroyo (S'11) was born in Barcelona, Spain. He received the M.S. degree in telecommunications engineering in $2011(\mathrm{BSc}+5)$ and the M.S. in Research on Information and Communication Technologies in $2012(\mathrm{MSc}+2)$, both from the Universitat Politècnica de CatalunyaBarcelonaTech. He is currently working toward the Ph.D. degree in GNSS-Reflectometry, with the Passive Remote Sensing Group, Department of Signal Theory and Communications, at the Universitat Politècnica de Catalunya-BarcelonaTech.

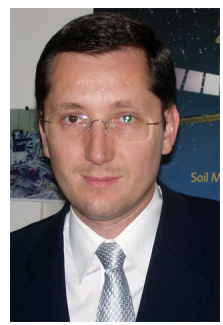

Adriano Camps (S'91-A'97-M'00-SM'03-F'11) was born in Barcelona, Spain, in 1969. He received the degree in telecommunications engineering and $\mathrm{Ph} . \mathrm{D}$. degree in telecommunications engineering from the Universitat Politècnica de Catalunya (UPC), Barcelona, Spain, in 1992 and 1996, respectively. In 1991 to 1992, he was at the ENS des Télécommunications de Bretagne, France, with an Erasmus Fellowship. Since 1993, he has been with the Electromagnetics and Photonics Engineering Group, Department of Signal Theory and Communications, UPC, where he was first Assistant Professor, Associate Professor in 1997, and Full Professor since 2007. In 1999, he was on sabbatical leave at the Microwave Remote Sensing Laboratory, of the University of Massachusetts, Amherst. Since 1993, he has been deeply involved in the European Space Agency SMOS Earth Explorer Mission, and since 2001 on the use of GNSS-R techniques to perform the sea state correction needed to retrieve salinity from radiometric observations. He has received a number of awards for his research and teaching activies, among which the Research Distinction of the Generalitat de Catalunya (2002) for contributions to microwave passive remote sensing; the European Young Investigator Award (2004) of the European Science Foundation, the ICREA Academia award (2009), and the 1st (2000) and 7th (2010) Duran Farell Awards. He has published more than 125 peer-reviewed journal papers, and more than 250 international conference presentations.

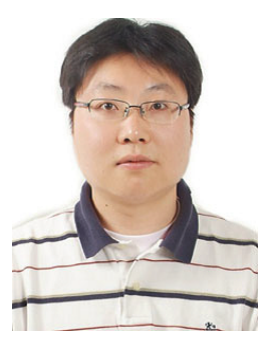

Hyuk Park (S'05-AM'09-M'12) was born in South Korea. He received the B.S. degree in mechanical engineering from the Korea Advanced Institute of Science and Technology (KAIST) in 2001, and received the M.S. and Ph.D. degree in information and mechatronics from the Gwangju Institute of Science and Technology (GIST), Korea, in 2003 and 2009, respectively. His main research interest is in the area of remote sensing, especially passive microwave remote sensing, including system design, modeling and simulation, and image processing. In 2009, he joined the remote sensing group of the Polytechnic University of Catalonia (UPC), Barcelona, as a postdoctoral researcher. He is a grant holder of NRF funded by Korean government in 2011. From 2012, he has been working as a research associate with Grant of Juan de la Cierva funded by Spanish Ministry of Economy and Competitiveness. Currently, he is working with the passive remote sensing group in the UPC for satellite remote sensing for microwave radiometry and GNSS-R.

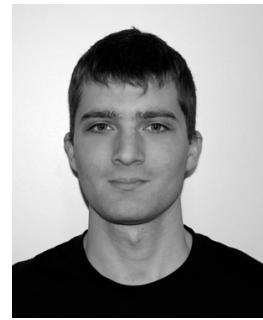

Daniel Pascual (S'11) was born in Barcelona, Spain, in 1985. He received the $\mathrm{BSc}+5$ degree in telecommunications engineering specialized in communications in 2011, and the MSc+2 degree in Research on Information and Communication Technologies in 2014, both from the Universitat Politècnica de Catalunya (UPC), Barcelona, Spain. In 2011 he joined the Passive Remote Sensing Group from UPC where he is currently working toward the $\mathrm{PhD}$ degree in GNSS-Reflectometry focused in ocean altimetry.

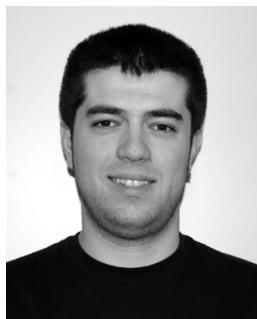

Raul Onrubia (S'10) was born in Barcelona, Spain $\mathrm{He}$ has received the M.S. degree in telecommunications engineering and is currently taking the M.S. in Research on Information and Communication Technologies at Universitat Politécnica de Catalunya. He is focused on RF systems and hardware developing in the GNSS-Reflectometry in the Passive Remote Sensing Group at the Signal Theory and Communications Department.

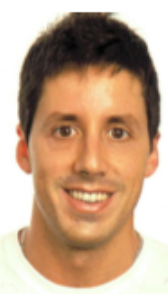

Francisco Martín (S'11) was born in Gandía (Valencia), Spain. He received the M.S. degree in telecommunications engineering in 2008 from the Universidad Politécnica de Valencia. In 2008, he was with the European Space Research Institute (ESRIN), European Space Agency (ESA), Frascati. From 2009-2011, he was with Programa Català d'Observació de la Terra (PCOT). He is currently working toward the Ph.D. degree in GNSSreflectometry, with the Passive Remote Sensing Group, Department of Signal Theory and Communications, at the Universitat Politècnica de Catalunya-Barcelona Tech. His PhD is under the framework of the NPI program offered by the European Space Agency (ESA). 